\title{
An exploration on magneto-shape memory effect in a Co-Ni single crystal
}

\author{
B. Jiang, Y. Liu, W. Zhou and X. Qi
}

Open Laboratory of Education, Ministry for High-Temperature Materials and Testing, Shanghai Jiao Tong University, Shanghai 200030, China

\begin{abstract}
A Co-33\%Ni (in mass) single crystal was grown by floating zone method in argon gas. The intrinsic magnetic properties, martensitic and austenitic transformation temperatures and Curie point of the crystal both in [001] and [111] directions were determined by the vibrating sample magnetometer (VSM) and magnetization balance (MB). The magnetic-field-induced strains were measured by strain gauges through the KJC magnetic measuring system. In [001] direction, the as-grown single crystal exhibits a high saturation magnetization of 124 $\mathrm{Am}^{2} \cdot \mathrm{kg}^{-1}$, which is nearly doubled compared with that of $\mathrm{Ni}_{2} \mathrm{MnGa}$. A reversible strain of $3 \%$ was induced by an applied pulse magnetic field of $2 \mathrm{~T}$ along [001] direction at a temperature (180K) in the range between $M_{s}$ and $M_{d}$, that is ten times more than that of $\mathrm{Ni}_{2} \mathrm{MnGa}$. While applying the pulsed magnetic field at temperatures below $\mathrm{M}_{\mathrm{s}}$ (such as $155 \mathrm{~K}$ and $145 \mathrm{~K}$ ), a recoverable field induced strain of $1 \%$ was obtained and about $0.5 \%$ strain was retained. After heating to room temperature, about $60 \%$ whole strain was recovered. If applying magnetic field along [111] direction of the crystal, no visible strain could be induced. According to the martensitic transformation theory on FCC to HCP structure, a mechanism based on the movement of $a / 6<112>$ partial dislocations driven by an applied magnetic field is presented.
\end{abstract}

\section{INTRODUCTION}

From the point of view of functional materials, piezoelectric ceramics and magneto-strictive materials exhibit rapid response, but their output strains are very small. Shape memory alloys, however, can develop strains of several percent, but the response is slow due to the thermal control (especially in the cooling run), which limits their applicability. The Magneto-Shape-Memory (MSM) alloy is a new kind of functional material, which exhibits both the characteristics of large output strains, as shape memory alloys: and of rapid response as piezoelectric ceramics and magneto-strictive materials. MSM alloys could be used in many high technology fields, such as actuator, sensor, micro-robot, fuel injector, active vibration and noise control system etc. Furthermore many new applications may be developed in the future due to their special properties. Hence, MSM alloys have become one of the most interesting research topics in the solid state field of physics and materials science and some improvement has been made for the past few years [1-5].

The typical MSM material is $\mathrm{Ni}_{2} \mathrm{MnGa}$. A magnetic-field-induced free strain of nearly $0.2 \%$ along [001] in unstressed crystals of $\mathrm{Ni}_{2} \mathrm{MnGa}$ with magnetic field of $8 \mathrm{kOe}$ applied at $265 \mathrm{~K}$ was firstly reported by Ullakko et al [1]. Subsequently James and Wuttig have observed that a non-stoichiometry 
single crystal of $\mathrm{Ni}_{2} \mathrm{MnGa}$ shows a magnetic-field-induced strain of $1.3 \%$ below $273 \mathrm{~K}$ [2]. Recently an output strain of $0.31 \%$ induced by an applied magnetic field of $6 \mathrm{kOe}$ at room temperature in a non-stoichiometry single crystal of $\mathrm{Ni}_{2} \mathrm{MnGa}$ has been reported [3]. On the basis of the research proceeding of $\mathrm{Ni}_{2} 22$

${ }_{2} \mathrm{MnGa}$, however, is a kind of intermetallic compound. The high brittleness is its own intrinsic disadvantage and it is difficult to get practical applications for its drawbacks. Therefore, to develop new kinds of MSM materials beyond $\mathrm{Ni}_{2} \mathrm{MnGa}$, which shows obvious MSM effect, is also an interesting subject to the physicists and materials scientists.

Here we report a primary study on a new MSM single crystal of Co-33\%Ni (in mass), which exhibits a great magnetic-field-induced strain in [001] direction by an applied pulse magnetic field of $2 \mathrm{~T}$. The possible mechanism is presented.

\section{EXPERIMENTAL PROCEDURE}

A Co-33\% Ni (in mass) single crystal was grown by Optical Floating Zone Furnace without crucible in purified argon gas. The starting material for the single crystal was prepared from metal elements Co and $\mathrm{Ni}$ with the purity of $99.95 \%$. Regular strips can be observed found in its surface by naked eye. Dislocations and stacking faults were observed by TEM.

The as-grown single crystal with a size of $\varnothing 8 \times 32 \mathrm{~mm}$ was heat-treated at $1173 \mathrm{~K}$ for 24 hours within purified argon gas and then was quenched into ice water. It was oriented by both X-ray back reflection Laue diffraction and $\mathrm{X}$-ray single crystal orientation instrument. The orientation results clearly show that [111] is the direction of crystal growth. Some samples were cut to $2 \times 2 \times 4 \mathrm{~mm}$ along [001] and [111] for intrinsic magnetic measurements by JKC magnetic measuring system, vibrating sample magnetometer (VSM) and magnetization balance (MB). Samples of $1 \times 4 \times 10 \mathrm{~mm}$ pieces with the longest axes parallel to [001] and [111] direction respectively were used for the strain measuring by strain gauges. The strain gauges, made by metal foil were attached on the broad surface of the specimens and the strain measuring directions were aligned with the longest axes. The critical temperatures, such as $M_{s}, M_{f}, A_{s}, A_{f}$ and Curie point $\left(T_{c}\right)$, were determined by ac susceptibility and magnetization measurements.

\section{RESULTS AND DISCUSSIONS}

Figure 1(a) shows the $\chi-\mathrm{T}$ curve, the ac susceptibility as a function of temperature from 90 to $290 \mathrm{~K}$, measured by $\mathrm{JKC}$ magnetic measuring system in [001] direction. The ac susceptibility gradually increases on decreasing the temperature. When the martensitic transformation begins, the ac susceptibility reduces sharply with an kink. In the cooling run the starting temperature, $M_{s}$ and the finishing temperature, $M_{f}$ of the martensitic transformation can be determined respectively. In the heating run, however, the critical temperatures of the reverse transformation can-not be determined because of the temperature limitation of this measuring system, where temperature range is only from $80 \mathrm{~K}$ to $373 \mathrm{~K}$. Therefore a magnetization balance method was adopted and the M-T curve measured in [001] direction from 10 to $900{ }^{\circ} \mathrm{C}$ was obtained as shown in Figure 1 (b). From this curve, the austenitic transformation temperatures $\left(A_{s}, A_{f}\right)$ and the Curie point $\left(T_{0}\right)$ can be determined. The ac susceptibility and the magnetization balance in [111] direction were also measured in the same way, and we find that the critical temperatures have no relations with the directions of this single crystal. 


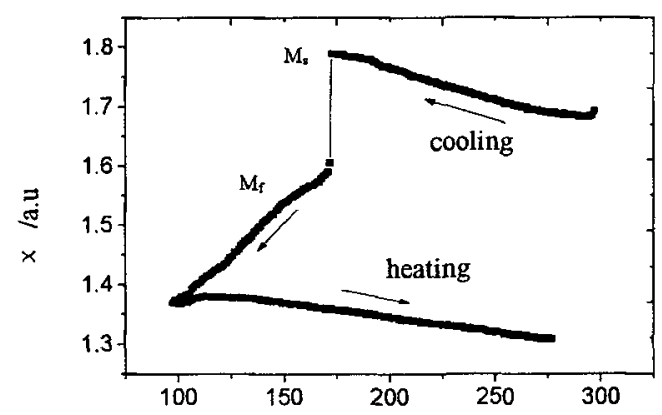

Temperature $/ K$

(a)

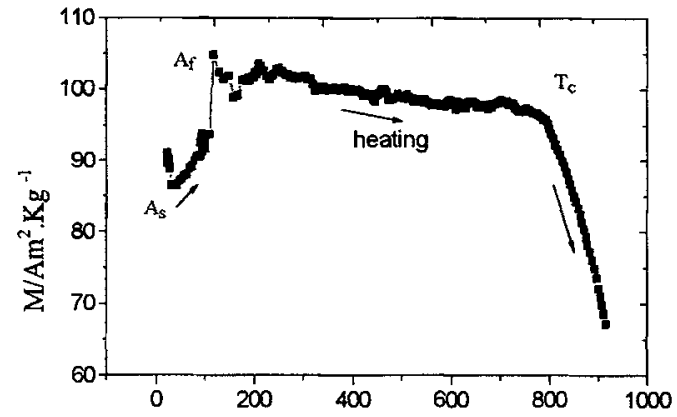

Temperature $/{ }^{\circ} \mathrm{C}$

(b)

Figure 1: The ac susceptibility vs. temperature, $\chi-T$ curve (a) and magnetization vs. temperature, M-T curve (b) in [001] direction

Figure 2 shows the magnetization vs. the magnetic field in [001] direction measured by VSM. The parent phase at high temperature exhibits a high saturation magnetization of $124 \mathrm{Am}^{2} \mathrm{~kg}^{-1}$, which is about twice when compared with that of the $\mathrm{Ni}_{2} \mathrm{MnGa}\left(66 \mathrm{Am}^{2} \cdot \mathrm{kg}^{-1}\right)$ [6]. The magnetization of the martensitic phase at lower temperature is also higher $\left(110 \mathrm{Am}^{2} \cdot \mathrm{kg}^{-1}\right)$. A high saturation value is very important for the magnetic induced martensitic transformation due to the high driving energy. Hence, from this point of view, Co-Ni alloy is a kind of potential MSM material with a large magnetic-field-induced strain.

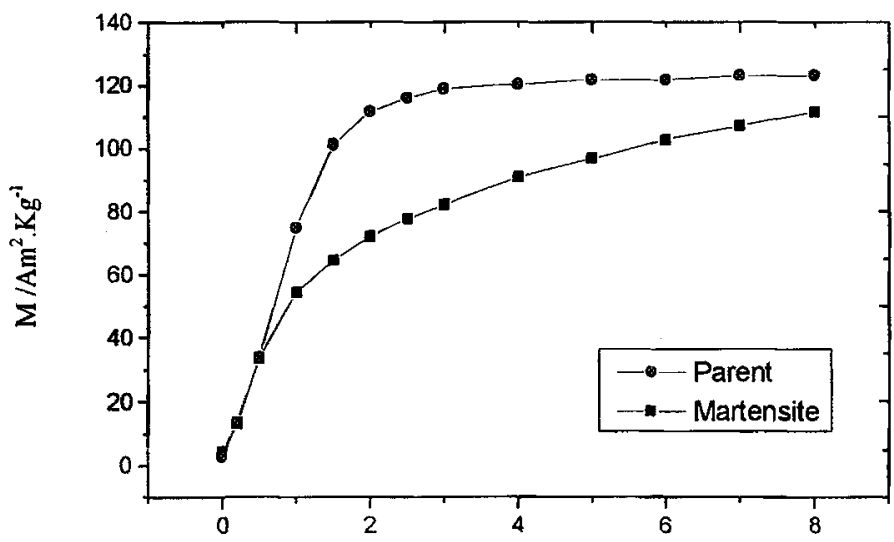

Magnetic field $/ \mathrm{kOe}$

Figure 2: The M-H curves of different phases in [001] direction 
Figure 3 shows the strain measured with discontinuous pulse magnetic field of $2 \mathrm{~T}$ applied along [001] direction during cooling and heating runs at different temperatures $240,228,212,200,190,180,155,145$ and $130 \mathrm{~K}$ etc. shown by the arrows. According to the data of reference [7], the $\mathrm{M}_{d}$ temperature of Co-33 $\% \mathrm{Ni}$ (in mass) is estimated between $190 \sim 200 \mathrm{~K}$. By applying a pulsed magnetic field at temperatures above $\mathrm{M}_{\mathrm{d}}$, for example at $228 \mathrm{~K}(\mathrm{BC})$, a drastic irreversible strain is induced due to the plastic deformation contributed by the slip of perfect dislocations of $a / 2<110>$. This is proved by the fact that a large amount of slip lines is observed on the surface of the specimen. It is well known that the direction of easy magnetization in Co-Ni single crystals is the $<111>$ direction. When an external magnetic field is applied along [001] direction, the element aligned in the [111] direction tends to turn to the external magnetic field direction, resulting in a shear stress applied to the specimen. The effect is analogous to the application of an external unidirectional stress. When the pulsed magnetic field is applied at 180K (below $\mathrm{M}_{\mathrm{d}}$ ) a reversible strain of $3 \%(\mathrm{DE})$ is induced, that is ten times more than that of $\mathrm{Ni}_{2} \mathrm{MnGa}$. The reason may be related to the generation of stress-induced $\varepsilon$-martensite by the movement of $a / 6<112>$ partial dislocations. The martensitic transformation occurs at $174.8 \mathrm{~K}$ resulting a shrinkage strain of about $2 \%$ (FG). At temperatures below $\mathrm{M}_{\mathrm{s}}$ (such as $155 \mathrm{~K}$ and $145 \mathrm{~K}$ ), the recoverable field-induced strain of $1 \%$ (HI) is obtained, which may result from the reorientation of the martensite variants through the movement of the interface boundaries between variants. At the same time about $0.5 \%$ strain is retained. After heating to room temperature, about $60 \%$ (JK) whole strain was recovered. From this curve, the martensitic transformation temperatures also can be determined as $\mathrm{M}_{\mathrm{s}} 170.6 \mathrm{~K}$ and $\mathrm{M}_{\mathrm{f}} 162 \mathrm{~K}$, which are coincident with that measured by VSM and MB.

Such a measurement is also done along [111] direction, but no visible strain can be found. Because the [111] direction is already the easy magnetization direction and no driving force builds up in this direction under external magnetic field .

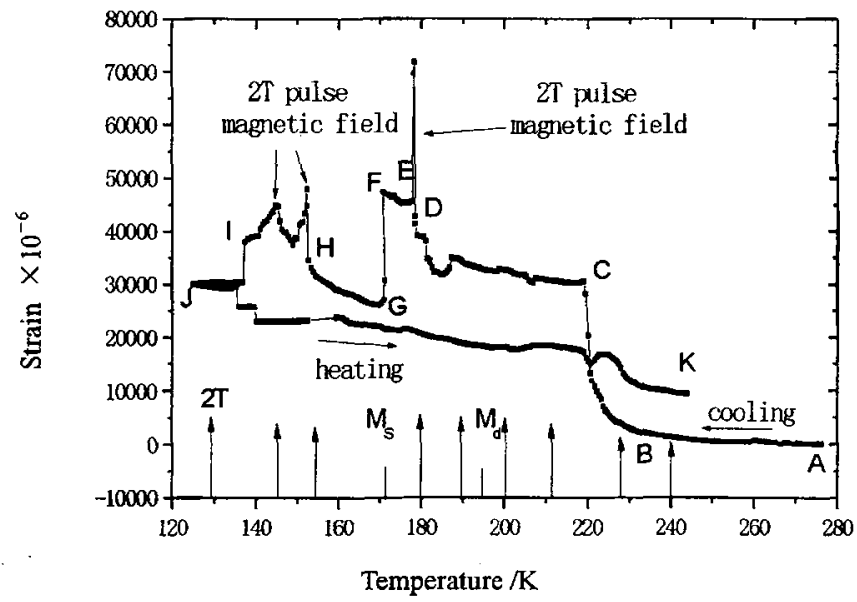

Figure 3: The strain vs. temperature with $2 \mathrm{~T}$ magnetic pulse field along [001] direction 


\section{SUMMARY}

Summarized from our experiments, several primary results are obtained as follows:

(1) A reversible strain of $3 \%$ is induced by applied pulse magnetic field of $2 \mathrm{~T}$ along [001] direction at a temperature of $180 \mathrm{~K}$ in the temperature between $\mathrm{M}_{d}$ and $\mathrm{M}_{\mathrm{s}}$.

(2) While applying the pulse magnetic field at temperatures below $M_{s}$ (such as $155 \mathrm{~K}$ and $145 \mathrm{~K}$ ), a recoverable field induced strain of $1 \%$ is obtained. At the same time about $0.5 \%$ strain is retained.

(3) After heating to room temperature, about $60 \%$ of the whole strain was recovered

(4) When the magnetic field is applied along [111] direction of the crystal, no measurable strain could be induced.

\section{Acknowledgments}

This work is supported by National Natural Science Foundation, P. R. China. Authors would like to thank Prof. T. Saburi and T. Kakeshita of Osaka University for preparing of single crystal specimen and to thank Prof. G. H. Wu of the National Lab of Magnetics in China for the help of magnetic measurements.

\section{References}

1. K. Ullakko, J. K. Huang, C. Kantner, R.C. O'Handley and V. V. Kokorin, Appl. Phys. Lett. 69(13), 1966 (1996).

2. R. D. James and M. Wuttig (personal communication from S. J. Murray, M. Farinelli, C. Kantner, J. K. Huang, S. M. Allen and R.C. O'Handley), J. of Applied Physics, 83 (11), 7297 (1998).

3. G. H. Wu, C. H. Yu, L. Q. Meng, F. M. Yang, et al, Appl. Phys. Lett. 75 (19), 6951 (1999).

4. K. Ullakko, J. K. Huang, V. V. Kokorin and R.C. O'Handley, Scr. Mater. 36, 1133 (1997).

5. R. C. O'Handley, J. Appl. Phys., 83, 3263(1998).

6. P. J. Webster/, K.R.A. Ziebeck, S. L. Town and M. S. Peak, Philos. Mag. B., 49, 295(1984).

7. J. B. Hess and C. S. Barrett, Trans., AIME., 194, 1664(1952). 